\title{
SUPERCONVERGENCE OF FINITE ELEMENT METHOD FOR PARABOLIC PROBLEM
}

\author{
DO Y. KWAK, SUNGYUN LEE, and QIAN LI
}

(Received 22 October 1998)

\begin{abstract}
We study superconvergence of a semi-discrete finite element scheme for parabolic problem. Our new scheme is based on introducing different approximation of initial condition. First, we give a superconvergence of $u_{h}-R_{h} u$, then use a postprocessing to improve the accuracy to higher order.
\end{abstract}

Keywords and phrases. Superconvergence, parabolic problem, postprocessing.

2000 Mathematics Subject Classification. Primary 65N15; Secondary 65N30.

1. Introduction. We consider the following parabolic problem:

$$
\begin{gathered}
u_{t}-\Delta u=f \quad \text { in } \Omega, \text { for } t>0, \\
u=0 \quad \text { on } \partial \Omega, \text { for } t \geq 0, \\
u(\cdot, 0)=v \quad \text { in } \Omega,
\end{gathered}
$$

where $\Omega \subset \mathbb{R}^{2}$ is a domain with smooth boundary. Suppose we are given a family $\mathbb{C}_{h}$ of quasi-uniform triangulation of $\Omega$, whose maximum diameter is denoted by $h$. Let $S_{h} \subset H_{0}^{1}(\Omega)$ be a standard finite element space consisting of continuous, piecewise polynomial of degree $k$. Define an elliptic projection $R_{h}: H_{0}^{1}(\Omega) \rightarrow S_{h}$ by

$$
\left(\nabla\left(R_{h} w-w\right), \nabla \chi\right)=0 \quad \forall \chi \in S_{h} .
$$

We consider the following map $u_{h}(t):[0, T] \rightarrow S_{h}$ defined by

$$
\left(u_{h, t}, \chi\right)+\left(\nabla u_{h}, \nabla \chi\right)=(f, x), \quad u_{h}(0)=v_{h},
$$

where $v_{h}$ is determined by

$$
\left(\nabla v_{h}, \nabla \chi\right)=(f(0), \chi)-\left(R_{h} u_{t}(0), \chi\right) \quad \forall \chi \in S_{h},
$$

and $u_{t}(0)$ is determined by (1.1). Superconvergence of finite element for parabolic problem has been studied by many authors. For example, Thomeé [8], Chen and Huang [1] studied superconvergence of the gradient in $L^{2}$ norm while Thomeé et al. [9] studied maximum norm superconvergence of gradient for linear finite element. Superconvergence of the lumped finite element method for linear and nonlinear parabolic problems were studied in [2] and [6], respectively. In this paper, we introduce a different way of approximating the initial condition, namely (1.4) and investigate the superconvergence of finite element for parabolic problem using any order element. 
To do so, we decompose the error as $u_{h}-u=u_{h}-R_{h} u+R_{h} u-u=\theta+\rho$ and estimate $\theta$ in a superconvergent order. Next, a postprocessing technique used in $[4,5]$ is employed to obtain higher order convergence. The rest of the paper is organized as follows. In Section 2, we show $\theta$ in $L^{2}$ and $H^{1}$ norm when $k>1$. For $\theta$, the superconvergence in $L^{\infty}$ and $W^{1, \infty}$ norm are also considered. In Section 3, the case $k=1$ is considered. The superconvergence of $\theta_{t}$ in $H^{1}$ and $\theta$ in $W^{1, \infty}$ norm are shown. In Section $4, W^{l, p}, l=0,1,(2<p<\infty)$ norm estimates are shown. Finally, in Section 5, we give some applications of the results obtained in Sections 2, 3 and 4. For example, a postprocessing technique is employed to obtain second-order superconvergence for gradient and first-order for the solution when $k>1$. First-order superconvergence is shown when $k=1$.

2. Superconvergence in $L^{2}, H^{1}, L^{\infty}$, and $W^{1, \infty}$ norm. We recall $\rho=R_{h} u-u$ and $\theta=u_{h}-R_{h} u$.

LEMMA 2.1. Let $1<p<\infty,(1 / p)+\left(1 / p^{\prime}\right)=1$. Then for any $g \in W^{1, p^{\prime}}(\Omega)$, we have, for $k>1$,

$$
\left|\left(D_{t}^{s} \rho, g\right)\right| \leq C h^{k+2}\left\|D_{t}^{s} u\right\|_{k+1, p}\|g\|_{1, p^{\prime}}
$$

where $D_{t}^{s}=\partial^{s} / \partial t^{s}$.

Proof. It suffices to prove the case for $s=0$. From standard finite element theory,

$$
\|\rho\|_{1, p} \leq C h^{k}\|u\|_{k+1, p}
$$

Consider the dual problem: given $g \in L^{p}(\Omega)$, find $w \in W^{3, p^{\prime}}(\Omega) \cap W_{0}^{1, p^{\prime}}(\Omega)$ satisfying

$$
\begin{gathered}
(\nabla v, \nabla w)=(g, v), \quad \forall v \in H_{0}^{1}(\Omega), \\
\|w\|_{3, p^{\prime}} \leq C\|g\|_{1, p^{\prime}} .
\end{gathered}
$$

Let $\prod_{h}$ denote the $S_{h}$ interpolation operator. Then by (2.3), (1.2), (2.2), (2.4), and the property of interpolation, we have

$$
\begin{aligned}
(g, \rho) & =(\nabla \rho, \nabla w)=\left|\left(\nabla \rho, \nabla\left(w-\Pi_{h} w\right)\right)\right| \\
& \leq\|\rho\|_{1, p}\left\|w-\Pi_{h} w\right\|_{1, p^{\prime}} \leq C h^{k}\|u\|_{k+1, p} h^{2}\|w\|_{3, p^{\prime}} \\
& \leq C h^{k+2}\|u\|_{k+1, p}\|g\|_{1, p^{\prime}} .
\end{aligned}
$$

LEMMA 2.2. We have

(i) $\theta_{t}(0)=0$, i.e., $u_{h, t}(0)=R_{h} u_{t}(0)$.

(ii) $\|\theta(0)\|_{1} \leq C h^{k+2}\left\|u_{t}(0)\right\|_{k+1}$.

Proof. From (1.4) and (1.3),

$$
\left(R_{h} u_{t}(0), \chi\right)=(f(0), \chi)-\left(\nabla v_{h}, \nabla \chi\right)=\left(u_{h, t}(0), \chi\right), \quad \chi \in S_{h} .
$$

Hence $R_{h} u_{t}(0)=u_{h, t}(0)$. For (ii), we see from (1.1),

$$
\left(u_{t}, v\right)+(\nabla u, \nabla v)=(f, v) .
$$


Subtraction of (1.3) from (2.7), and noting (1.2), give

$$
\left(\theta_{t}, \mathcal{X}\right)+(\nabla \theta, \nabla \mathcal{X})=-\left(\rho_{t}, \mathcal{X}\right), \quad \chi \in S_{h} .
$$

Set $t=0$ and noting that $\theta_{t}(0)=0$, we have

$$
(\nabla \theta(0), \nabla \chi)=-\left(\rho_{t}(0), \chi\right)
$$

Take $\chi=\theta(0)$ in (2.9). Then we see from Lemma 2.1,

$$
\|\nabla \theta(0)\|^{2}=\left|\left(\rho_{t}(0), \theta(0)\right)\right| \leq C h^{k+2}\left\|u_{t}(0)\right\|_{k+1}\|\theta(0)\|_{1} .
$$

Since $|\cdot|$ and $\|\cdot\|$ are equivalent in $H_{0}^{1}(\Omega)$,

$$
\|\theta(0)\|_{1} \leq C\|\nabla \theta(0)\| \leq C h^{k+2}\left\|u_{t}\right\|_{k+1} .
$$

THEOREM 2.3. We have first-order superconvergence for $\left\|\theta_{t}\right\|$ and second-order superconvergence for $\left\|\nabla \theta_{t}\right\|$. In other words,

$$
\left\|\theta_{t}(t)\right\|+\left(\int_{0}^{t}\left\|\nabla \theta_{t}\right\|^{2} d \tau\right)^{1 / 2} \leq C h^{k+2}\left(\int_{0}^{t}\left\|u_{t t}\right\|_{k+1}^{2} d \tau\right)^{1 / 2}
$$

holds.

Proof. Differentiating error equation (2.8),

$$
\left(\theta_{t t}, \chi\right)+\left(\nabla \theta_{t}, \nabla \chi\right)=-\left(\rho_{t t}, \chi\right), \quad \chi \in S_{h} .
$$

Take $x=\theta_{t}$. Then by Lemma 2.1, we have

$$
\begin{aligned}
\frac{1}{2} \frac{d}{d t}\left\|\theta_{t}\right\|^{2}+\left\|\nabla \theta_{t}\right\|^{2} & =\left|\left(\rho_{t t}, \theta_{t}\right)\right| \leq C h^{k+2}\left\|u_{t t}\right\|_{k+1}\left\|\theta_{t}\right\|_{1} \\
& \leq C h^{2(k+2)}\left\|u_{t t}\right\|_{k+1}^{2}+\frac{1}{2}\left\|\nabla \theta_{t}\right\|^{2},
\end{aligned}
$$

where arithmetic-geometric inequality was used in the last line. Elimination of $(1 / 2)\left\|\nabla \theta_{t}\right\|^{2}$ and integration, give, by Lemma 2.2(i),

$$
\begin{aligned}
\left\|\theta_{t}(t)\right\|^{2}+\int_{0}^{t}\left\|\nabla \theta_{t}(\tau)\right\|^{2} d \tau & \leq\left\|\theta_{t}(0)\right\|^{2}+C h^{2(k+2)} \int_{0}^{t}\left\|u_{t t}(\tau)\right\|_{k+1}^{2} d \tau \\
& \leq C h^{2(k+2)} \int_{0}^{t}\left\|u_{t t}(\tau)\right\|_{k+1}^{2} d \tau .
\end{aligned}
$$

THEOREM 2.4. We have second-order superconvergence for $\left\|\theta_{t}\right\|_{1}$ and first-order for $\left\|\theta_{t t}\right\|$,

$$
\left(\int_{0}^{t}\left\|\theta_{t t}\right\|^{2} d \tau\right)^{1 / 2}+\left\|\theta_{t}(t)\right\|_{1} \leq C h^{k+2}\left[\left\|u_{t t}(t)\right\|_{k+1}+\left(\int_{0}^{t}\left\|u_{t t t}\right\|_{k+1}^{2} d \tau\right)^{1 / 2}\right] .
$$


Proof. From (2.13) with $\chi=\theta_{t t}$,

$$
\left\|\theta_{t t}\right\|^{2}+\frac{1}{2} \frac{d}{d t}\left\|\nabla \theta_{t}\right\|^{2}=-\left(\rho_{t t}, \theta_{t t}\right) .
$$

Integration, and noting that $\theta_{t}(0)=0$, gives

$$
\begin{aligned}
\int_{0}^{t}\left\|\theta_{t t}\right\|^{2} d \tau+\frac{1}{2}\left\|\nabla \theta_{t}\right\|^{2} & =-\int_{0}^{t}\left(\rho_{t t}, \theta_{t t}\right) d \tau \\
& =-\left.\left(\rho_{t t}, \theta_{t}\right)\right|_{0} ^{t}+\int_{0}^{t}\left(\rho_{t t t}, \theta_{t}\right) d \tau \\
& =-\left(\rho_{t t}, \theta_{t}\right)+\int_{0}^{t}\left(\rho_{t t t}, \theta_{t}\right) d \tau
\end{aligned}
$$

Using Lemma 2.1, left-hand side of (2.18) is

$$
\begin{aligned}
& \leq C h^{k+2}\left\|u_{t t}\right\|_{k+1}\left\|\theta_{t}\right\|_{1}+C h^{k+2} \int_{0}^{t}\left\|u_{t t t}\right\|_{k+1}\left\|\theta_{t}\right\|_{1} d \tau \\
& \leq C h^{2(k+2)}\left\|u_{t t}\right\|_{k+1}^{2}+\frac{1}{4}\left\|\theta_{t}\right\|_{1}^{2}+C h^{2(k+2)} \int_{0}^{t}\left\|u_{t t t}\right\|_{k+1}^{2} d \tau+C \int_{0}^{t}\left\|\theta_{t}\right\|_{1}^{2} d \tau .
\end{aligned}
$$

Elimination of (1/4) $\left\|\theta_{t}\right\|_{1}^{2}$ and usage of Gronwall inequality give (2.16).

THEOREM 2.5. We have second-order superconvergence for $\|\theta\|_{1}$.

$$
\|\theta(t)\|_{1} \leq C h^{k+2}\left[\left\|u_{t}(0)\right\|_{k+1}+\left(\int_{0}^{t}\left\|u_{t t}\right\|_{k+1}^{2} d \tau\right)^{1 / 2}\right] .
$$

Proof. By Lemma 2.2 and Theorem 2.3, we have

$$
\begin{aligned}
\|\theta(t)\|_{1} & \leq\|\theta(0)\|_{1}+\int_{0}^{t}\left\|\theta_{t}\right\|_{1} d \tau \\
& \leq\|\theta(0)\|_{1}+C\left(\int_{0}^{t}\left\|\theta_{t}\right\|_{1}^{2} d \tau\right)^{1 / 2} \\
& \leq C h^{k+2}\left\|u_{t}(0)\right\|_{k+1}+C h^{k+2}\left(\int_{0}^{t}\left\|u_{t t}\right\|_{k+1}^{2} d \tau\right)^{1 / 2} .
\end{aligned}
$$

THEOREM 2.6. We have first-order superconvergence for $\|\theta\|$.

$$
\|\theta(t)\| \leq C h^{k+2}\left[\left\|u_{t}(0)\right\|_{k+1}+\left(\int_{0}^{t}\left\|u_{t}\right\|_{k+1}^{2} d \tau\right)^{1 / 2}\right] .
$$

PRoof. Recall that error equation (2.8)

$$
\left(\theta_{t}, \chi\right)+(\nabla \theta, \nabla \chi)=-\left(\rho_{t}, \chi\right)
$$

Take $\chi=\theta$ in (2.8). Then we see from Lemma 2.1,

$$
\begin{aligned}
\frac{1}{2} \frac{d}{d t}\|\theta(t)\|^{2}+\|\nabla \theta\|^{2} & =-\left(\rho_{t}, \theta\right) \leq C h^{k+2}\left\|u_{t}\right\|_{k+1}\|\theta\|_{1} \\
& \leq C h^{2(k+2)}\left\|u_{t}\right\|_{k+1}^{2}+\|\nabla \theta\|^{2} .
\end{aligned}
$$


Elimination of $\|\nabla \theta\|^{2}$ and integration, give, by Lemma 2.2,

$$
\begin{aligned}
\|\theta(t)\|^{2} & \leq\|\theta(0)\|^{2}+c h^{2(k+2)} \int_{0}^{t}\left\|u_{t}\right\|_{k+1}^{2} d \tau \\
& \leq C h^{2(k+2)}\left\|u_{t}(0)\right\|_{k+1}^{2}+c h^{2(k+2)} \int_{0}^{t}\left\|u_{t}\right\|_{k+1}^{2} d \tau .
\end{aligned}
$$

Now we study $L^{\infty}, W^{1, \infty}$ superconvergence. First we need Green's functions. The discrete Green's function $G_{h}^{z} \in S_{h}$ for $z \in \Omega$ is defined by

$$
\left(\nabla G_{h}^{z}, \nabla \chi\right)=\chi(z), \quad \chi \in S_{h}
$$

The derivative type Green's function $g_{h, i}^{z} \in S_{h},(i=1,2)$ is defined by

$$
\left(\nabla g_{h, i}^{z}, \nabla \chi\right)=\frac{\partial}{\partial x_{i}} \chi(z), \quad \chi \in S_{h}
$$

Green's functions posses the following properties (see $[9,10])$.

LEMMA 2.7. We have

$$
\begin{gathered}
\left\|G_{h}^{z}\right\|+\left\|G_{h}^{z}\right\|_{1, p^{\prime}} \leq C, \quad 1 \leq p^{\prime}<2, \\
\left\|g_{h, i}^{z}\right\|^{2}+\left\|g_{h, i}^{z}\right\|_{1,1} \leq C \log \frac{1}{h} .
\end{gathered}
$$

THEOREM 2.8. We have the following estimate:

$$
\|\theta(t)\|_{0, \infty} \leq C h^{k+2}\left[\left\|u_{t}(t)\right\|_{k+1, p}+\left(\int_{0}^{t}\left\|u_{t t}\right\|_{k+1}^{2} d \tau\right)^{1 / 2}\right], \quad p>2 .
$$

Proof. By taking $\chi=\theta$ in the definition (2.26), we have by (2.8), Lemmas 2.1, 2.7, and Theorem 2.3,

$$
\begin{aligned}
|\theta(z, t)| & =\left|\left(\nabla G_{h}^{z}, \nabla \theta\right)\right|=\left|\left(\rho_{t}, G_{h}^{z}\right)+\left(\theta_{t}, G_{h}^{z}\right)\right| \\
& \leq C h^{k+2}\left\|u_{t}\right\|_{k+1, p}\left\|G_{h}^{z}\right\|_{1, p^{\prime}}+\left\|\theta_{t}\right\|\left\|G_{h}^{z}\right\| \\
& \leq C h^{k+2}\left\|u_{t}\right\|_{k+1, p}+C h^{k+2}\left(\int_{0}^{t}\left\|u_{t t}\right\|_{k+1}^{2} d \tau\right)^{1 / 2} .
\end{aligned}
$$

Now take supremum over all $z \in \Omega$.

THEOREM 2.9. We have the following estimate:

$$
\|\theta(t)\|_{1, \infty} \leq C h^{k+2-\epsilon}\left[\left\|u_{t}\right\|_{k+1, p}+\left(\int_{0}^{t}\left\|u_{t t}\right\|_{k+1}^{2} d \tau\right)^{1 / 2}\right],
$$

for any $\epsilon>2 / p, p<\infty$ large enough. 
Proof. For $z \in \Omega$, we see from (2.27), (2.8), Lemma 2.7, and Theorem 2.3,

$$
\begin{aligned}
\left|\frac{\partial}{\partial x_{i}} \theta(z)\right| & =\left|\left(\nabla g_{h, i}^{z}, \nabla \theta\right)\right|=\left|\left(\rho_{t}, g_{h, i}^{z}\right)+\left(\theta_{t}, g_{h, i}^{z}\right)\right| \\
& \leq C h^{k+2}\left\|u_{t}\right\|_{k+1, p}\left\|g_{h, i}^{z}\right\|_{1, p^{\prime}}+\left\|\theta _ { t } \left|\left\|\mid g_{h, i}^{z}\right\|\right.\right. \\
& \leq C h^{k+2-2 / p}\left\|u_{t}\right\|_{k+1, p}\left\|g_{h, i}^{z}\right\|_{1,1}+C h^{k+2}\left(\int_{0}^{t}\left\|u_{t t}\right\|_{k+1}^{2} d \tau\right)^{1 / 2}\left\|g_{h, i}^{z}\right\| \\
& \leq C h^{k+2-\epsilon}\left(\int_{0}^{t}\left\|u_{t t}\right\|_{k+1}^{2} d \tau\right)^{1 / 2}\left\|g_{h, i}^{z}\right\|,
\end{aligned}
$$

where inverse estimate

$$
\left\|g_{h, i}^{z}\right\|_{1, p^{\prime}} \leq C h^{-2 / p}\left\|g_{h, i}^{z}\right\|_{1,1}, \quad 1 \leq p^{\prime}<2,2<p \leq \infty
$$

was used in the second inequality.

3. The case $k=1$. Here the corresponding finite element space $S_{h}$ is a linear finite element space. We make suitable modification of Lemma 2.2 to obtain the following lemma.

LEMMA 3.1.

$$
\|\theta(0)\|_{1} \leq C h^{2}\left\|u_{t}(0)\right\|_{2} .
$$

Proof. We recall (2.9)

$$
(\nabla \theta(0), \nabla \chi)=-\left(\rho_{t}(0), \chi\right), \quad x \in S_{h}
$$

Take $\chi=\theta(0)$. Then, we see that

$$
\|\nabla \theta(0)\|^{2}=\left|\left(\rho_{t}(0), \chi(0)\right)\right| \leq\left\|\rho_{t}(0)\right\| \cdot\|\theta(0)\| \leq C h^{2}\left\|u_{t}(0)\right\|_{2} \cdot\|\nabla \theta(0)\| .
$$

THEOREM 3.2. We have

$$
\left\|\theta_{t}(t)\right\|+\left(\int_{0}^{t}\left\|\nabla \theta_{t}\right\|^{2} d \tau\right)^{1 / 2} \leq C h^{2}\left(\int_{0}^{t}\left\|u_{t t}\right\|_{2}^{2} d \tau\right)^{1 / 2}
$$

Proof. We recall (2.13)

$$
\left(\theta_{t t}, \chi\right)+\left(\nabla \theta_{t}, \nabla \chi\right)=-\left(\rho_{t t}, \chi\right), \quad \chi \in S_{h} .
$$

Taking $\chi=\theta_{t}$, we see that

$$
\begin{aligned}
\frac{1}{2} \frac{d}{d t}\left\|\theta_{t}\right\|^{2}+\left\|\nabla \theta_{t}\right\|^{2} & \leq C\left\|\rho_{t t}\right\| \cdot\left\|\theta_{t}\right\| \\
& \leq C h^{2}\left\|u_{t t}\right\|_{2}\left\|\nabla \theta_{t}\right\| \\
& \leq C h^{4}\left\|u_{t t}\right\|_{2}^{2}+\frac{1}{2}\left\|\nabla \theta_{t}\right\|^{2} .
\end{aligned}
$$

Elimination of $(1 / 2)\left\|\nabla \theta_{t}\right\|^{2}$ and integration, give the result. 
COROLLARY 3.3. We have

$$
\|\theta(t)\|_{1} \leq C h^{2}\left(\int_{0}^{t}\left\|u_{t t}\right\|_{2}^{2} d \tau\right)^{1 / 2}
$$

THEOREM 3.4. We have

$$
\left(\int_{0}^{t}\left\|\theta_{t t}\right\|^{2} d \tau\right)^{1 / 2}+\left\|\theta_{t}(t)\right\|_{1} \leq C h^{2}\left[\left\|u_{t t}(t)\right\|_{2}+\left(\int_{0}^{t}\left\|u_{t t t}\right\|_{2}^{2} d \tau\right)^{1 / 2}\right] .
$$

Proof. Taking $x=\theta_{t t}$ in (2.13), we see that

$$
\left\|\theta_{t t}\right\|^{2}+\frac{1}{2} \frac{d}{d t}\left\|\nabla \theta_{t}\right\|^{2}=-\left(\rho_{t t}, \theta_{t t}\right) .
$$

Integrating and noting $\theta_{t}(0)=0$, we have

$$
\begin{aligned}
\int_{0}^{t}\left\|\theta_{t t}\right\|^{2} d \tau+\frac{1}{2}\left\|\nabla \theta_{t}\right\|^{2} & =-\int_{0}^{t}\left(\rho_{t t}, \theta_{t t}\right) d t \\
& =-\left(\rho_{t t}, \theta_{t}\right)+\int_{0}^{t}\left(\rho_{t t t}, \theta_{t}\right) d \tau \\
& \leq\left\|\rho_{t t}\right\| \cdot\left\|\theta_{t}\right\|+\int_{0}^{t}\left\|\rho_{t t t}\right\| \cdot\left\|\theta_{t}\right\| d \tau \\
& \leq C h^{2}\left\|u_{t t}\right\|_{2} \cdot\left\|\theta_{t}\right\|+c h^{2} \int_{0}^{t}\left\|u_{t t t}\right\|_{2} \cdot\left\|\theta_{t}\right\| d \tau \\
& \leq C h^{4}\left\|u_{t t}\right\|_{2}^{2}+\frac{1}{4}\left\|\nabla \theta_{t}\right\|^{2}+c h^{4} \int_{0}^{t}\left\|u_{t t t}\right\|_{2}^{2} d \tau+\int_{0}^{t}\left\|\nabla \theta_{t}\right\|^{2} d \tau .
\end{aligned}
$$

Now Gronwall inequality gives the result.

LEMMA 3.5. For $1<p<2$, we have the following estimate:

$$
\left\|\nabla g_{h, i}^{z}\right\|_{0, p} \leq C \quad \text { for } i=1,2 \text {. }
$$

Proof. Let $(1 / p)+\left(1 / p^{\prime}\right)=1$. For any $\phi \in L^{p^{\prime}}(\Omega)$, let $\Psi$ be the solution of

$$
-\Delta \Psi=\phi \quad \text { in } \Omega, \quad \Psi=0 \quad \text { on } \partial \Omega .
$$

Then we have

$$
\|\Psi\|_{2, p^{\prime}} \leq C\|\phi\|_{0, p^{\prime}}
$$

Setting $g_{h}=g_{h, i}^{z}$, we have, by (3.12), (1.2), and (2.27),

$$
\left(g_{h}, \phi\right)=\left(\nabla g_{h}, \nabla \Psi\right)=\left(\nabla g_{h}, \nabla R_{h} \Psi\right)=\frac{\partial}{\partial x_{i}} R_{h} \Psi(z) .
$$

Thus, we see from $W^{1, \infty}$ stability of $R_{h}$, imbedding theorem and (3.13) that

$$
\left(g_{h}, \phi\right) \leq\left\|R_{h} \Psi\right\|_{1, \infty} \leq C\|\Psi\|_{1, \infty} \leq C\|\Psi\|_{2, p^{\prime}} \leq C\|\phi\|_{0, p^{\prime}},
$$

we have

$$
\left\|g_{h}\right\|_{0, p}=\sup _{\phi \in L^{p^{\prime}(\Omega)}} \frac{\left(g_{h}, \phi\right)}{\|\phi\|_{0, p^{\prime}}} \leq C
$$


THEOREM 3.6. We have

$$
\|\theta(t)\|_{1, \infty} \leq C h^{2}\left[\left\|u_{t}(t)\right\|_{2, p}+\left\|u_{t t}(t)\right\|_{2}+\left(\int_{0}^{t}\left\|u_{t t t}\right\|_{2}^{2} d \tau\right)^{1 / 2}\right], \quad p>2 .
$$

Proof. Setting $X=g_{h, i}^{z}$ in (2.8), we obtain by (2.27), (3.11) and imbedding theorem, we have

$$
\begin{aligned}
\frac{\partial}{\partial x_{i}} \theta(z, t) & \leq\left(u_{t}-u_{h, t}, g_{h, i}^{z}\right) \\
& \leq\left(\left\|\rho_{t}\right\|_{0, p}+\left\|\theta_{t}\right\|_{0, p}\right)\left\|g_{h, i}^{z}\right\|_{0, p^{\prime}},(1 / p)+\left(1 / p^{\prime}\right)=1 \\
& \leq C\left(\left\|\rho_{t}\right\|_{0, p}+\left\|\theta_{t}\right\|_{1}\right) .
\end{aligned}
$$

By standard estimate, we have

$$
\left\|\rho_{t}\right\|_{0, p} \leq C h^{2}\left\|u_{t}\right\|_{2, p} .
$$

Combining (3.8), (3.19) with (3.18), we obtain the desired result.

COROLlary 3.7. We have

$$
\|\theta(t)\|_{0, \infty} \leq C h^{2}\left[\left\|u_{t}(t)\right\|_{2, p}+\left\|u_{t t}(t)\right\|_{2}+\left(\int_{0}^{t}\left\|u_{t t t}\right\|_{2}^{2} d \tau\right)^{1 / 2}\right], \quad p>2 .
$$

4. Superconvergence in $L^{p}$ and $W^{1, p},(2<p<\infty)$

THEOREM 4.1. We have

$$
\|\theta\|_{0, p} \leq C h^{k+2}\left[\left\|u_{t}(0)\right\|_{k+1}+\left(\int_{0}^{t}\left\|u_{t t}\right\|_{k+1}^{2} d \tau\right)^{1 / 2}\right], \quad k>1 .
$$

Proof. From Sobolev inequality, we have, for $2<p<\infty$,

$$
\|\chi\|_{0, p} \leq C\|\chi\|_{1}, \quad \chi \in S_{h} .
$$

The conclusion directly follows from Theorem 2.5 .

THEOREM 4.2. We have

$$
\begin{aligned}
& \|\theta(t)\|_{1, p} \leq C h^{k+2}\left[\left\|u_{t}(t)\right\|_{k+1, p}+\left(\int_{0}^{t}\left\|u_{t t}\right\|_{k+1}^{2} d \tau\right)^{1 / 2}\right], \quad k>1, \\
& \|\theta(t)\|_{1, p} \leq C h^{2}\left[\left\|u_{t}(t)\right\|_{2, p}+\left\|u_{t t}(t)\right\|_{2}+\left(\int_{0}^{t}\left\|u_{t t t}\right\|_{2}^{2} d \tau\right)^{1 / 2}\right], \quad k=1 .
\end{aligned}
$$

Proof. Let $p(2<p<\infty)$ and $p^{\prime}$ be conjugate indices, and let $\phi \in L^{p^{\prime}}(\Omega)$ with $\|\phi\|_{0, p^{\prime}}=1$ and $\phi_{x}$ be any component of $\nabla \phi$. If $\psi$ is the solution of

$$
(\nabla v, \nabla \psi)=-\left(\phi_{x}, v\right), \quad \forall v \in H_{0}^{1}(\Omega)
$$


with the regularity property [7]

$$
\|\psi\|_{1, p^{\prime}} \leq C_{p}\|\phi\|_{0, p^{\prime}}=C_{p} .
$$

Then by Green's formula, equations (4.5), (1.2), (2.8), Lemma 2.1, Theorem 2.3, Sobolev lemma, and (4.6), we have

$$
\begin{aligned}
\left(\theta_{x}, \phi\right) & =-\left(\phi_{x}, \theta\right)=(\nabla \theta, \nabla \psi)=\left(\nabla \theta, \nabla R_{h} \psi\right)=-\left(\rho_{t}, R_{h} \psi\right)-\left(\theta_{t}, R_{h} \psi\right) \\
& \leq C h^{k+2}\left\|u_{t}(t)\right\|_{k+1, p}\left\|R_{h} \psi\right\|_{1, p^{\prime}}+\left\|\theta_{t}(t)\right\|\left\|R_{h} \psi\right\| \\
& \leq C h^{k+2}\left[\left\|u_{t}(t)\right\|_{k+1, p}+\left(\int_{0}^{t}\left\|u_{t t}\right\|_{k+1}^{2} d \tau\right)^{1 / 2}\right]\left\|R_{h} \psi\right\|_{1, p^{\prime}} \\
& \leq C h^{k+2}\left[\left\|u_{t}(t)\right\|_{k+1, p}+\left(\int_{0}^{t}\left\|u_{t t}\right\|_{k+1}^{2} d \tau\right)^{1 / 2}\right] .
\end{aligned}
$$

Now noting that

$$
\left\|\theta_{x}\right\|_{0, p}=\sup _{\psi \in L^{p^{\prime}}(\Omega)}\left(\theta_{x}, \phi\right), \quad\|\phi\|_{0, p^{\prime}}=1,
$$

the conclusion (4.3) is obtained. To prove (4.4), we note that

$$
\|\theta\|_{1, p} \leq C\|\theta\|_{1, \infty} .
$$

This, together with (3.17), proves the theorem.

5. Application. We now give an application of the results derived in Sections 2 and 3.

As an example, let $T_{h}$ be a quasi-uniform rectangular partition of $\Omega \subset \mathbb{R}^{2}$ and let $S_{h}$ be the space of continuous piecewise polynomials

$$
S_{h}=\left\{v \in H_{0}^{1}(\Omega), v \in Q^{k}(\tau), \tau \in T_{h}\right\},
$$

where

$$
Q^{k}=\operatorname{span}\left\{x_{1}^{i} x_{2}^{j}, 0 \leq i, j \leq k\right\} .
$$

Introduce two kinds of operators (see [3, 4]), the vertices-edges-element interpolation $i_{h}^{k}$ and the high-interpolation operator $I_{2 h}^{k+l}(l=1,2)$. They satisfy the following properties:

$$
\begin{gathered}
\left\|u-I_{2 h}^{k+l} u\right\|_{m, p} \leq C h^{k+l+1-m}\|u\|_{k+l+1, p}, \quad 1 \leq k, m=0,1,(2 \leq p \leq \infty), l=1,2, \\
I_{2 h}^{k+l} i_{h}^{k}=I_{2 h}^{k+l}, \quad k \geq 1, l=1,2, \\
\left\|I_{2 h}^{k+l} x\right\|_{m, p} \leq C\|x\|_{m, p}, \quad \forall x \in S_{k}, 1 \leq k, m=0,1,(2 \leq p \leq \infty), l=1,2 .
\end{gathered}
$$

Using these properties we can improve global convergence from $k$-to $k+2$-order for gradient, and from $k+1$-to $k+2$-order for solution when $k \geq 2$. When $k=1$, we get one order gain for the gradient. 
THEOREM 5.1. For $k \geq 2$, we have the following results:

$$
\begin{aligned}
& \left\|u-I_{2 h}^{k+1} u_{h}\right\| \leq C h^{k+2}\left[\left\|u_{t}(0)\right\|_{k+1}+\left(\int_{0}^{t}\left\|u_{t}\right\|_{k+1}^{2} d \tau\right)^{1 / 2}+\|u(t)\|_{k+3}\right], \\
& \left\|u-I_{2 h}^{k+1} u_{h}\right\|_{0, p} \\
& \quad \leq C h^{k+2}\left[\left\|u_{t}(0)\right\|_{k+1}+\left(\int_{0}^{t}\left\|u_{t t}\right\|_{k+1}^{2} d \tau\right)^{1 / 2}+\|u(t)\|_{k+3, p}\right], \quad p>2, \\
& \left\|u_{t}-I_{2 h}^{k+1} u_{h, t}\right\| \leq C h^{k+2}\left[\left(\int_{0}^{t}\left\|u_{t t}\right\|_{k+1}^{2} d \tau\right)^{1 / 2}+\left\|u_{t}(t)\right\|_{k+3}\right], \\
& \left\|u-I_{2 h}^{k+1} u_{h}\right\|_{0, \infty} \\
& \quad \leq C h^{k+2}\left[\left\|u_{t}(t)\right\|_{k+1, p}+\left(\int_{0}^{t}\left\|u_{t t}\right\|_{k+1}^{2} d \tau\right)^{1 / 2}+\|u(t)\|_{k+3, \infty}\right], \quad p>2, \\
& \left\|u-I_{2 h}^{k+2} u_{h}\right\|_{1} \leq C h^{k+2}\left[\left\|u_{t}(0)\right\|_{k+1}+\left(\int_{0}^{t}\left\|u_{t t}\right\|_{k+1}^{2} d \tau\right)^{1 / 2}+\|u(t)\|_{k+3}\right] \\
& \left\|u-I_{2 h}^{k+2} u_{h}\right\|_{1, p} \\
& \quad \leq C h^{k+2}\left[\left\|u_{t}(t)\right\|_{k+1, p}+\left(\int_{0}^{t}\left\|u_{t t}\right\|_{k+1}^{2} d \tau\right)^{1 / 2}+\|u(t)\|_{k+3, p}\right], \quad p>2, \\
& \left\|u-I_{2 h}^{k+2} u_{h}\right\|_{1, \infty} \\
& \quad \leq C h^{k+2-\epsilon}\left[\left\|u_{t}(t)\right\|_{k+1, p}+\left(\int_{0}^{t}\left\|u_{t t}\right\|_{k+1}^{2} d \tau\right)^{1 / 2}+\|u(t)\|_{k+3, \infty}\right]
\end{aligned}
$$

for any $\epsilon>2 / p, p$ large enough,

$$
\left\|u_{t}-I_{2 h}^{k+2} u_{h, t}\right\|_{1} \leq C h^{k+2}\left[\left\|u_{t t}(t)\right\|_{k+1}+\left(\int_{0}^{t}\left\|u_{t t t}\right\|_{k+1}^{2} d \tau\right)^{1 / 2}+\|u(t)\|_{k+1}\right] .
$$

Proof. Obviously, by (5.4) and (5.5), we have

$$
\begin{gathered}
u-I_{2 h}^{k+l} u_{h}=u-I_{2 h}^{k+l} u+I_{2 h}^{k+l}\left(i_{h}^{k} u-R_{h} u\right)+I_{2 h}^{k+1}\left(R_{h} u-u_{h}\right), \\
\left\|u-I_{2 h}^{k+l} u_{h}\right\|_{m, p} \leq\left\|u-I_{2 h}^{k+l} u\right\|_{m, p}+C\left\|i_{h}^{k} u-R_{h} u\right\|_{m, p}+C\left\|R_{h} u-u_{h}\right\|_{m, p},
\end{gathered}
$$

for $l=1,2$. The estimates of first and third terms are shown in (5.3) and Theorems 2.6, $4.1,2.3,2.8,2.5,4.2,2.9$ and 2.4 (in this order). It remains estimate the second term. By [5, Corollary to Theorem 3.4.2],

$$
\left\|i_{h}^{k} u-R_{h} u\right\|_{m, p} \leq C h^{k+2}\|u\|_{k+3, p}, \quad 2 \leq p \leq \infty, m=0,1,
$$

so that

$$
\left\|i_{h}^{k} u_{t}-R_{h} u_{t}\right\|_{m, p} \leq C h^{k+2}\left\|u_{t}\right\|_{k+3, p} .
$$

Thus, the proof is complete. 
THEOREM 5.2. For $k=1$, we have

$$
\begin{aligned}
& \left\|u-I_{2 h}^{2} u_{h}\right\|_{1} \leq C h^{2}\left[\left(\int_{0}^{t}\left\|u_{t t}\right\|_{2}^{2} d \tau\right)^{1 / 2}+\|u(t)\|_{3}\right] \\
& \left\|u-I_{2 h}^{2} u_{h}\right\|_{1, p} \\
& \quad \leq C h^{2}\left[\left\|u_{t}(t)\right\|_{2, p}+\left\|u_{t t}\right\|_{2}+\left(\int_{0}^{t}\left\|u_{t t t}\right\|_{2}^{2} d \tau\right)^{1 / 2}+\|u(t)\|_{3, p}\right], \quad 2<p \leq \infty \\
& \left\|u_{t}-I_{2 h}^{2} u_{h, t}\right\|_{1} \leq C h^{2}\left[\left\|u_{t t}(t)\right\|_{2}+\left(\int_{0}^{t}\left\|u_{t t t}\right\|_{2}^{2} d \tau\right)^{1 / 2}+\left\|u_{t}(t)\right\|_{3}\right]
\end{aligned}
$$

Proof. When $k=1$ and $m=1$ in (5.15)

$$
\left\|u-I_{2 h}^{2} u_{h}\right\|_{1, p} \leq\left\|u-I_{2 h}^{2} u_{h}\right\|_{1, p}+C\left\|i_{h}^{2} u-R_{h} u\right\|_{1, p}+C\left\|R_{h} u-u_{h}\right\|_{1, p} .
$$

It suffices to estimate the second term. By [3], for any $\chi \in S_{h}$

$$
\begin{aligned}
\left(\nabla\left(i_{h}^{2} u-R_{h} u\right), \nabla \chi\right) & =\left(\nabla\left(i_{h}^{2} u-u\right), \nabla \chi\right) \\
& =O\left(h^{2}\right)\|u\|_{3, p}\|\chi\|_{1, p^{\prime}}, \quad \frac{1}{p}+\frac{1}{p^{\prime}}=1, p \geq 2 .
\end{aligned}
$$

Using the same method as in [4] we have

$$
\begin{gathered}
\left\|i_{h}^{2} u-R_{h} u\right\|_{1, p} \leq C h^{2}\|u\|_{3, p}, \\
\left\|i_{h}^{2} u_{t}-R_{h} u_{t}\right\|_{1, p} \leq C h^{2}\left\|u_{t}\right\|_{3, p} .
\end{gathered}
$$

These together with (3.7), (3.17), and (3.8) completes the proof.

ACKNOWLEDGEMENTS. D. Y. Kwak is partially supported by KOSEF under contract number 97-07-01-01-01-3. Q. Li is partially supported by KFSTS under Brain Pool Program.

\section{REFERENCES}

[1] C. M. Chen and Y. Q. Huang, High Accuracy Theory of Finite Element Methods, Hunan Science and Technology Press, China, 1995.

[2] C. M. Chen and V. Thomée, The lumped mass finite element method for a parabolic problem, J. Austral. Math. Soc. Ser. B 26 (1985), no. 3, 329-354. MR 86m:65117. Zbl 576.65110.

[3] Q. Lin, A rectangle test for finite element analysis, Proc. System Science and System Eng. (Hong Kong), Great Wall Culture Publ. Co., 1991, pp. 213-216.

[4] Q. Lin, N. N. Yang, and A. H. Zhou, A rectangle test for interpolated finite elements, Proc. System Science and System Eng. (Hong Kong), Great Wall Culture Publ. Co., 1991, pp. 217-229.

[5] Q. Lin and Q. D. Zhu, The Preprocessing and Postprocessing for the Finite Element Method, Shanghai Scientific and Technical Publishers, China, 1994.

[6] Y. Y. Nie and V. Thomée, A lumped mass finite-element method with quadrature for a nonlinear parabolic problem, IMA J. Numer. Anal. 5 (1985), no. 4, 371-396. MR 87b:65163. Zbl 591.65079.

[7] M. Schechter, On $L^{p}$ estimates and regularity. I, Amer. J. Math. 85 (1963), 1-13. MR 32\#6051. Zbl 113.30603. 
[8] V. Thomée, Galerkin Finite Element Methods for Parabolic Problems, Lecture Notes in Mathematics 1054, Springer-Verlag, Berlin, New York, 1984. MR 86k:65006. Zbl 528.65052.

[9] V. Thomée, J. C. Xu, and N. Y. Zhang, Superconvergence of the gradient in piecewise linear finite-element approximation to a parabolic problem, SIAM J. Numer. Anal. 26 (1989), no. 3, 553-573. MR 90e:65165. Zbl 678.65079.

[10] C. D. Zhu and Q. Lin, Youxianyuan Chaoshoulian Lilun [The hyerconvergence theory of finite elements], Hunan Science and Technology Publishing House, Changsha, 1989. MR 93j:65191.

KwaK: Department of MAthematics, KAIST, TAEJON, 305-701, Korea

E-mail address: dykwak@math.kaist.ac.kr

LeE: DePartment of MATHematics, KAIST, TAejon, 305-701, KoreA

E-mail address: sylee@mathx.kaist.ac.kr

Li: Department of Mathematics, Shandong Normal University, Jinan, Shandong, 250014, CHINA 


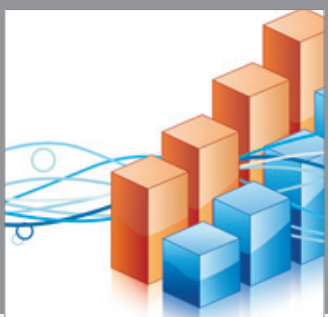

Advances in

Operations Research

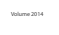

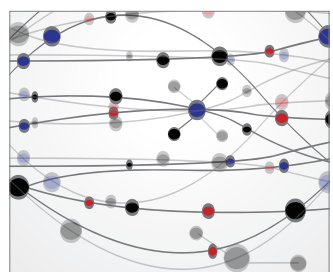

\section{The Scientific} World Journal
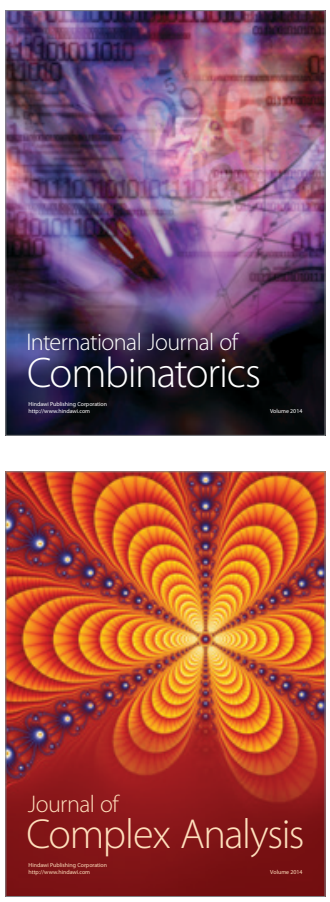

International Journal of

Mathematics and

Mathematical

Sciences
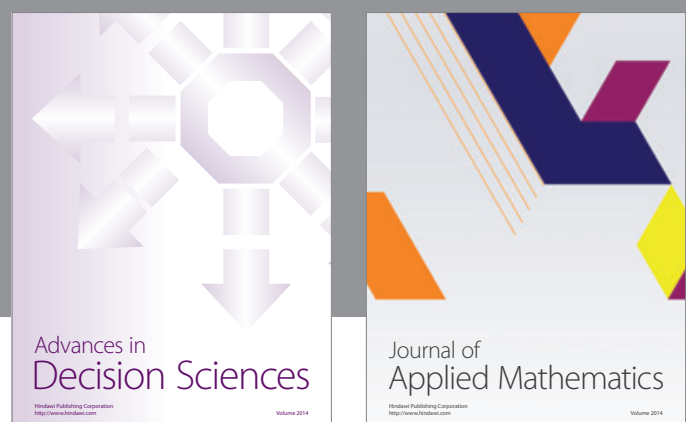

Journal of

Applied Mathematics
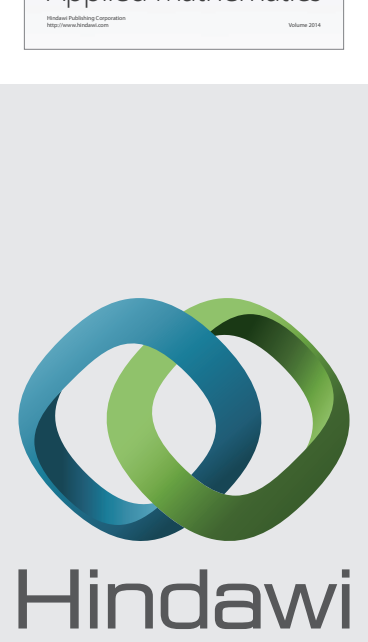

Submit your manuscripts at http://www.hindawi.com
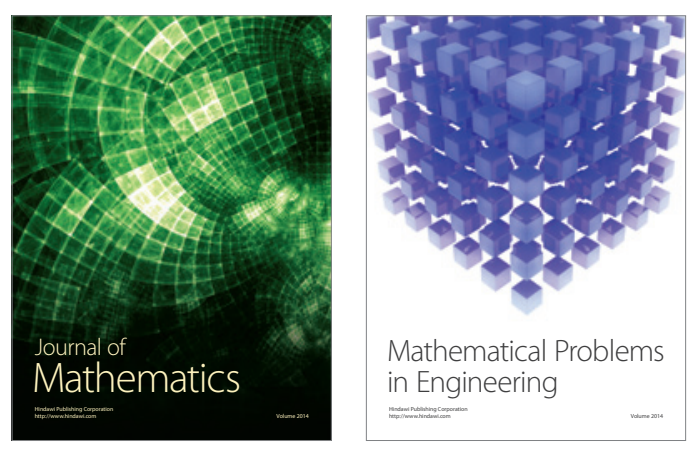

Mathematical Problems in Engineering
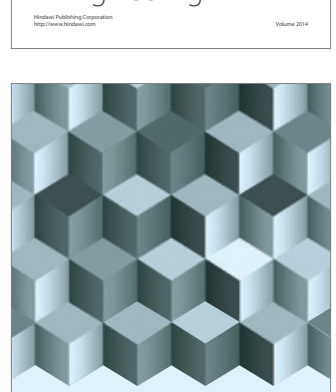

Journal of

Function Spaces
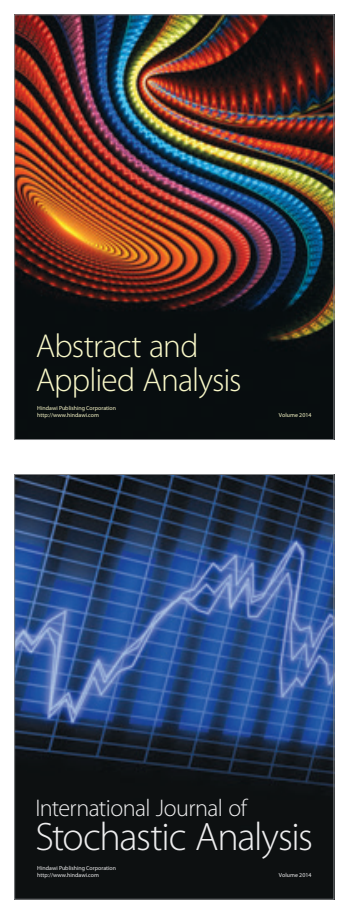

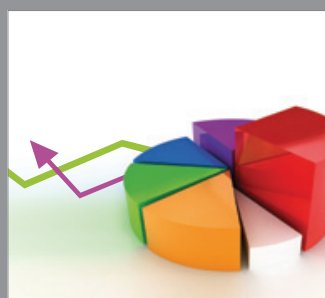

ournal of

Probability and Statistics

Promensencen
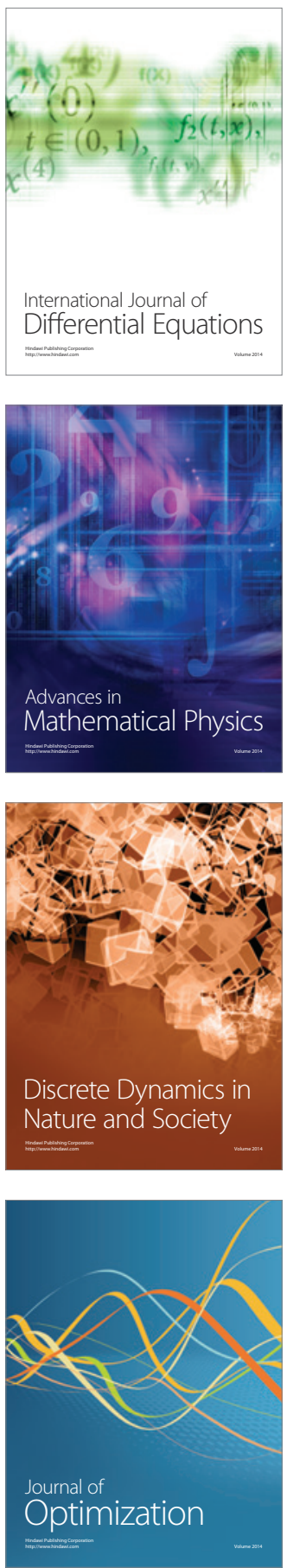